\title{
EXPERIENÊNCIA DE INTEGRAÇÃO DA EXPERIMENTAÇÃO REMOTA NO ENSINO DE FÍSICA DO ENSINO MÉDIO: PERCEPÇÃO DOS ALUNOS
}

\author{
Carine Heck - carine.heck@ gmail.com - UFSC \\ Karine dos Santos Coelho - kakascoelho@ hotmail.com - UFSC \\ José Pedro Schardosim Simão - josepedrosimao@gmail.com - UFSC \\ Isabela Nardi da Silva - isabela.nardi@ hotmail.com - UFSC \\ Juarez Bento da Silva - juarez.b.silva@ieee.org - UFSC \\ Simone Meister Sommer Bilessimo - simone.bilessimo@gmail.com - UFSC
}

Resumo: A presente pesquisa teve por objetivo identificar e analisar a percepção dos alunos do Ensino Médio acerca do uso e da integração da experimentação remota no ensino e aprendizagem de Física. A investigação foi desenvolvida na $3^{a}$ série do Ensino Médio na disciplina de Física de uma escola pública Estadual de Santa Catarina. A pesquisa foi precedida por um questionário que visava traçar o perfil tecnológico dos estudantes, e sucedida por outra que procurava verificar a opinião dos estudantes sobre experimentação remota e o impacto que estas tecnologias podem ter em sua educação por conta de proporcionarem aulas práticas. Na percepção dos alunos, a experimentação remota contribui para motivação no estudo de física e proporciona novas formas de aprender para além do quadro e giz.

Palavras-chave: Experimentação remota, ensino de física, educação básica

\section{REMOTE EXPERIMENTATION IN PHYSICS TEACHING IN HIGH SCHOOL INTEGRATION EXPERIENCE: STUDENTS' PERCEPTION}

\begin{abstract}
This research verify the viability of using the remote lab "DC Electric Panel", developed at the Laboratory $\mathrm{X}$ on physics teaching. The survey was preceded by a questionnaire that aimed to outline the technological profile of the students, and succeeded by another that sought to verify the views of students on remote experimentation and the impact these technologies can have on their education due to provide practical classes. The study proved to be satisfactory, since the results were positive and valid, and the work will provide research in the future.
\end{abstract}

Keywords: Remote experimentation, physics teaching, high school

\section{Introdução}

O ensino de Física no Brasil tem sido praticado repetidamente por meio da apresentação de conceitos, leis, fórmulas e resolução de exercícios, que pouco se relacionam com a realidade dos alunos. As mudanças sociais e culturais estão abrindo caminhos para novas formas de aprender e ensinar para além do quadro e giz. "Os alunos da educação científica precisam não tanto de mais informação (embora possam também precisar disso), mas, sobretudo de capacidade de organizá-la e interpretá-la, para lhe dar sentido" (POZO \& CRESPO, 2009, P.24). Segundo Moreira e Penido (2009) é preciso inserir a experimentação como uma parte integrante como método de investigação, uma vez que a formação do pensamento crítico e investigativo dos alunos se dá por meio de atividades práticas nas aulas de física. As aulas laboratoriais aproximam o aluno do seu cotidiano, melhoram a compreensão da teoria e desenvolvem 
habilidades como manusear instrumentos aguçando a sua curiosidade, o que leva a uma aprendizagem mais significativa.

Entretanto, há muitas barreiras impedindo a realização das atividades experimentais nas aulas de física, entre elas, destacam-se: falta de tempo para preparação de plano de aula e conteúdo didático que relaciona teoria e prática; falta de pessoal para manutenção acabando por limitar o acesso a equipamentos mais delicados; falta de equipamentos laboratoriais e/ou materiais; laboratórios com dimensões não adequadas ao número de alunos e ausência de laboratórios presenciais na escola. Conforme dados do Censo escolar 2014 (QEdu, 2016), apenas 11\% das escolas brasileiras dispõem de laboratórios físicos. Vale destacar que este número insignificante de laboratórios existentes conta com todas as dificuldades mencionadas acima.

Atualmente uma maneira de suprir a falta de laboratórios presenciais nas escolas públicas de educação básica é a utilização de Laboratório de Experimentação Remota móvel. Este laboratório proporciona a realização e controle em tempo real dos experimentos, usando como meio computador e internet, onde o aluno poderá acessar e utilizar o experimento a qualquer hora, em qualquer lugar e realizar o experimento quantas vezes for necessário. Os laboratórios acessados remotamente possibilitam aos alunos uma "aproximação deste com o mundo real, (...) e também representam uma maneira de compartilhamento de recursos, de tal forma a reduzir os custos para utilização destes recursos, por parte das instituições de ensino, além de constituir um fator de enriquecimento da experiência educacional" (PALADINI, 2008, p.5). "Os laboratórios remotos para práticas buscam resolver de uma forma efetiva e prática os problemas de acesso aos laboratórios clássicos" (SILVA, 2006, p.121).

Nesse caminho a experimentação remota por meio de dispositivos móveis ou convencionais com acesso a internet, tem se mostrado um instrumento privilegiado para a escola contemporânea. Visto que, "a presença expressiva dos dispositivos móveis na vida das pessoas tem alterado de forma significativa os estilos de vida da sociedade, em particular dos jovens" (SILVA, 2006, p.129).

O painel "Quadro Elétrico CC" oportuniza estudar e demonstrar circuitos resistivos simples, aplicar a Lei de Ohm e Leis de Kirchhoff, além de trabalhar a associação de resistores. Este trabalho descreve a tecnologia utilizada na construção do "Painel Elétrico CC", relata um modelo de aplicação em sala de aula e também traz resultados de uma aplicação em uma escola pública de educação básica.

Portanto, este trabalho tem por objetivo analisar a viabilidade do experimento remota "Quadro Elétrico CC", no processo de ensino e aprendizagem de física na Educação Básica (Ensino Médio), este experimento encontra-se disponível no link: http://relle.ufsc.br/labs/1, e foi desenvolvido pelo Laboratório de Experimentação Remota - Rexlab,. Neste contexto a tecnologia é utilizada, não apenas como mero instrumento de informação, mas possibilita a compreensão e interpretação do conhecimento científico e tecnológico por acesso virtual, permitindo ao aluno, observar, analisar e interpretar dados reais.

\section{Experimento Remoto Painel Elétrico CC}

O experimento foi desenvolvido utilizando hardware e software aberto, a partir de uma arquitetura modular, com o intuito de facilitar a replicação e compartilhamento de recursos. Esta arquitetura, apresentada na Figura 1, é dividida em três módulos Interface de Usuário (UI), WebServer e Experimento Remoto, e se repete em outros experimentos remotos desenvolvidos no Laboratório Rexlab. $\mathrm{O}$ acesso ao laboratório remoto é feito utilizando apenas computador com acesso a internet, pelo link: http://gtmre.ufsc.br/moodle/, sendo que qualquer pessoa pode fazer o acesso. 


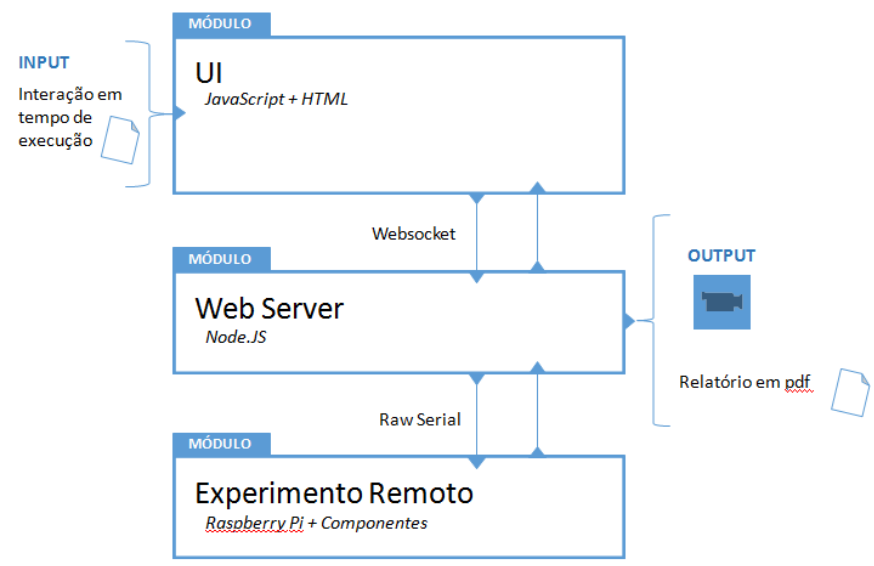

Figura 1 - Diagrama de arquitetura do experimento remoto Painel Elétrico CC

O circuito controlado remotamente pelo usuário está disposto em um painel de acrílico, e é composto por um conjunto de sete (7) resistores e sete (7) chaves que permitem a obtenção de diferentes combinações. Além disso, sete (7) módulos relê permitem o acionamento das chaves, e três (3) voltímetros junto a sete (7) amperímetros fazem a leitura de tensão e corrente. Estes componentes estão conectados à uma placa de controle e aquisição, que é responsável por obter os dados das leituras dos sensores e transmitir os comandos enviados pelo usuário aos atuadores.

Um RaspberryPi B+ executando Raspbian, uma distribuição Linux baseada em Debian, é utilizado como servidor web. O servidor roda uma aplicação Node.js que provê um conjunto de operações para manipulação do experimento. Uma webcam genérica captura o vídeo da execução do experimento, que é então transmitido em formato MJPEG por meio da aplicação de código aberto Motion, utilizada para gerenciamento de câmeras de vigilância.

$\mathrm{O}$ acesso ao experimento é feito a partir de uma página web de layout responsivo, com o intuito de proporcionar ao usuário uma experiência semelhante de acesso em telas de dispositivos de diferentes tamanhos. A interface foi desenvolvida utilizando HTML5, utilizando o framework JavaScritpjQuery e o framework CSS Bootstrap.

Ao acessar a página do experimento (Figura 2), o usuário tem informações sobre o assunto abordado, duração da sessão e um código para incorporar o experimento em outras páginas. Se o experimento estiver sendo utilizado por outro usuário, o usuário terá de aguardar até o fim da sessão ou até que o outro usuário saia do experimento para obter acesso.

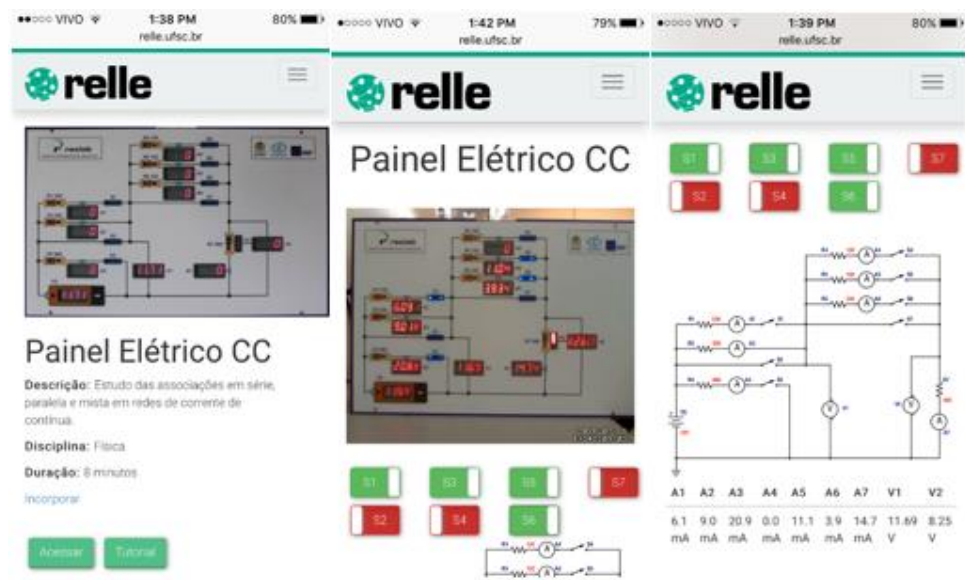

Figura 2 - Interface da página de acesso ao experimento 
Durante sua sessão, o tem acesso à interface de manipulação do experimento, onde ao clicar nas chaves na página, um comando é enviado ao experimento para que ligue ou desligue esta chave. O usuário pode observar no streaming de vídeo as chaves ligadas ou desligadas e as leituras dos voltímetros e amperímetros.

O laboratório de experimentação remota é um sistema, combinado de um computador (servidor) e dispositivos externos reais (experimentos) que se encontram num laboratório físico (SCHUHMACHER ET AL. 2005). Com acesso a internet e um computador ou dispositivo móvel (tablet, smartphone, etc..) o usuário (aluno, professor, etc...) pode controlar via Internet, os experimentos reais que se encontram neste laboratório, de qualquer lugar a qualquer hora, como se o usuário estivesse no local, onde se encontra os experimentos. Isto permite ao usuário não só o acesso livre a eles, como também a interação direta com os experimentos, além da obtenção online de informações e resultados reais dos procedimentos realizados. Diferente da "simulação que envolve a criação de modelos dinâmicos e simplificados do mundo real, os quais procuram se aproximar da realidade, para que se consiga estudar os processos reais de forma mais confortável e previsível" (KHALIL, 2012, p2).

Portanto, o laboratório remoto encontra-se fisicamente separado do aluno, mas acessando um experimento remoto este aluno irá controlar instrumentos e dispositivos reais, além de visualizar os fenômenos e obter resultados precisos e não aproximados como em uma simulação que muitas vezes desconsidera as variações do ambiente.

\section{Metodologia}

Este trabalho tem uma abordagem investigativa e de acordo com sua natureza de dados, é do tipo qualitativa. Segundo Freire (2013), este tipo de pesquisa respeita a experiência de cada indivíduo participante da pesquisa. Esta é uma pesquisa de análise mais profunda e, portanto se caracteriza como um estudo de casso de acordo. Utiliza-se o estudo de caso, "quando se quer identificar a aplicabilidade de uma teoria, suas limitações ou as diferenças entre a teoria e a prática" (FREIRE, 2013, p.57).

$\mathrm{O}$ estudo contou com a colaboração de alunos do $3^{\circ}$ ano do ensino médio de uma escola da rede pública localizada no litoral da região sul do país. É uma escola estadual com aproximadamente 850 alunos, onde os recursos para mantê-la provêm do governo do Estado de Santa Catarina.

A aplicação iniciou-se no último bimestre do ano letivo, onde os alunos trabalharam em grupos para utilizarem o experimento seguindo um roteiro elaborado pela professora da classe. Este roteiro continha questões que necessitavam o acesso direto ao experimento e questões que utilizavam somente a imagem ilustrativa que se encontra no próprio site. Esta foi uma forma que a professora encontrou de não deixar os alunos sem atividade na sala de aula. Uma vez que o experimento só pode ser acessado por uma pessoa ou grupo. Visto que este ainda é um protótipo.

O tema proposto para trabalhar o experimento remoto "Painel Elétrico CC" foi associação de resistores em série, paralela e mista.

Quadro 1 - Roteiro do experimento remoto: painel elétrico CC ROTEIRO EXPERIMENTO REMOTO: PAINEL ELÉTRICO CC

1. Explique o que significa as informações abaixo:

$\mathrm{A} 1, \mathrm{~A} 2, \mathrm{~A} 3, \mathrm{~A} 4, \mathrm{~A} 5, \mathrm{~A} 6, \mathrm{~A} 7=$

R1, R2, R3, R4, R5, R6, R7=

$\mathrm{V} 1, \mathrm{~V} 2=$ 
$\mathrm{S} 1, \mathrm{~S} 2, \mathrm{~S} 3, \mathrm{~S} 4, \mathrm{~S} 5, \mathrm{~S} 6, \mathrm{~S} 7=$

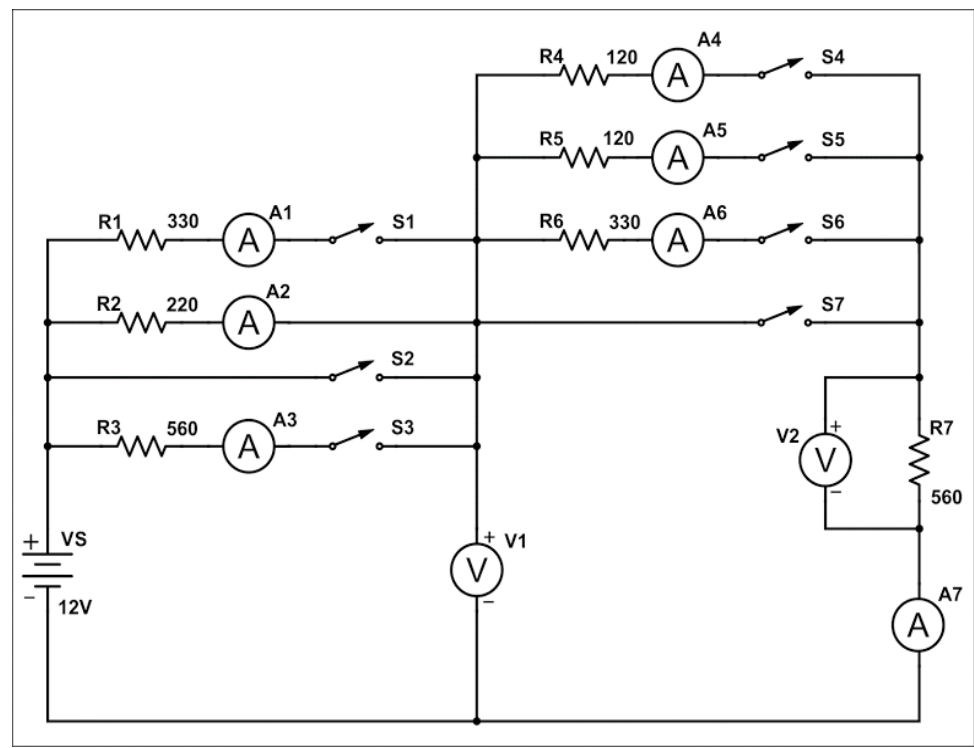

Figura 3 - Esquema quadro elétrico CC

Fonte: autores

2. O circuito é abastecido por corrente contínua ou alternada? Justifique.

Acessar o experimento remoto. Abra as chaves 1, 2, 3 e 7, fechando as chaves 4, 5, e 6 .

3. Em quais amperímetros ocorre a passagem de corrente elétrica?

4. Qual a intensidade de corrente elétrica registrada nos amperímetros de acordo com a reposta da questão acima?

5. Nos fios onde estão acomodados os amperímetros A4, A5 e A6 também estão acoplados resistores, respectivamente, R4, R5 e R6. O que você observou na relação entre a distribuição da corrente elétrica nos três fios com os valores dos resistores?

6. Represente a associação de resistores descrita nessa operação.

7. Qual é o nome da associação operada?

Acessar o experimento remoto, fechando as chaves 1 e 7 e abrindo as chaves 2, 3, 4, 5, e 6 .

8. Em quais amperímetros ocorre a passagem de corrente elétrica? Quais são os valores de corrente elétrica registrada nesses amperímetros?

9. Por que a intensidade de corrente elétrica no amperímetro A2 é maior do que no amperímetro A1?

10. Represente a associação de resistores descrita nessa operação.

11. Qual é o nome da associação operada?

Acessar o experimento remoto, fechando as chaves 4 e 6 e abrindo as chaves 1, 2, 3, 5 e 7 .

12. Em quais amperímetros ocorre a passagem de corrente elétrica? Quais são os valores de corrente elétrica registrada nesses amperímetros?

13. Por que a intensidade de corrente elétrica no amperímetro A2 é maior do que nos amperímetros A4 e A6?

14. Efetuando-se a adição das correntes dos amperímetros A4 e A6, o que pode-se 
constatar em relação ao valor com o amperímetro A2?

15. Qual relação pode-se estabelecer entre os valores dos amperímetros A4 e A6 com os resistores acomodados nos fios?

16. Represente a associação de resistores descrita nessa operação.

17. Qual é o nome da associação operada?

18. Quais exemplos do dia a dia usam essas associações de resistores com fonte de corrente contínua? Cite alguns exemplos.

Antes de iniciar o estudo foi aplicado um questionário visando traçar o perfil tecnológico dos alunos, e após a realização do procedimento experimental a professora disponibilizou um questionário visando verificar a opinião dos estudantes sobre as qualidades percebidas dos laboratórios remotos e promover a reflexão dos mesmos sobre o potencial destas tecnologias na sua aprendizagem e habilidades através de aulas práticas.

É importante traçar o perfil tecnológico dos alunos, uma vez que, este traz elementos que podem contribuir para o entendimento do estudo.

Os dados foram coletados através de um questionário disponibilizado e respondido no Ambiente Virtual de Aprendizagem (Moodle), no ano de 2015 por 40 estudantes com faixa etária entre 16 a 20 anos do $3^{\circ}$ ano do ensino médio.

Em relação a inclusão digital dos estudantes pesquisou-se a disponibilidade da tecnologia. O quadro 2 mostra um percentual significativo de $85 \%$, tanto para disponibilidade de computador como para acesso a internet. Este resultado mostra a importância de um planejamento para a integração da tecnologia na sala de aula, uma vez que os $15 \%$ que não possuem computador e nem acesso a internet, teriam que utilizar os equipamentos tecnológicos da própria escola.

Quadro 2 - Disponibilidade de computador e conexão com a internet.

\begin{tabular}{|l|l|l|}
\hline & Sim & Não \\
\hline Possui computador & $85 \%$ & $15 \%$ \\
\hline Possui acesso à internet & $85 \%$ & $15 \%$ \\
\hline
\end{tabular}

Quanto ao meio preferencial de acesso a internet, os alunos responderam que utilizam computadores ou dispositivos móveis com $65 \%$ para os dispositivos móveis, pois a grande maioria destes alunos possui um aparelho de celular e estes estão sempre presente, seja na escola ou em qualquer outro ambiente. Apenas 35\% utilizam o computador para acessar a internet. Com relação ao local preferencial de acesso $90 \%$ dos estudantes preferem a residência para se conectar a internet, visto que a escola não disponibiliza o sinal de internet sem fio. Os $10 \%$ que preferem acessar a internet da escola utilizam as salas informatizadas e os computadores de mesa.

\section{Resultados e análise}

Nesta seção apresentam-se os dados obtidos no questionário após o uso do experimento remoto "Painel Elétrico CC" na disciplina de física. O objetivo deste estudo foi analisar a viabilidade da experimentação remota "Quadro Elétrico CC" por meio da opinião dos estudantes sobre as qualidades percebidas dos laboratórios remotos e promover a reflexão dos mesmos sobre o potencial desta tecnologia na sua aprendizagem e habilidades através de aulas práticas. 
O questionário foi disponibilizado e respondido no Ambiente Virtual de Aprendizagem - Moodle. As questões são no formato da Escala de Likert, composta com 11 questões. O questionário é formado por diversos elementos em forma de afirmações, sobre os quais deve ser expresso seu grau de concordância e decida se concorda totalmente (CT) concordar em parte (CP), desaprovo totalmente (DT), desaprovo em parte (DP), neutro $(\mathrm{N})$.

A frequência observada para cada opção do questionário das onze questões está sistematizada no quadro $3 \mathrm{em}$ forma de porcentagem.

Quadro 3 - Grau de concordância em relação à experiência quanto a utilização do experimento remoto "Painel Elétrico CC".

\begin{tabular}{|c|c|c|c|c|c|}
\hline Questão & $\mathbf{C T}$ & $\mathbf{C P}$ & $\mathbf{N}$ & DP & DT \\
\hline $\begin{array}{l}\text { 1.A possibilidade de visualizar e controlar os } \\
\text { experimentos remotos de qualquer lugar é um fator } \\
\text { importante. }\end{array}$ & $76 \%$ & $24 \%$ & 0 & 0 & 0 \\
\hline $\begin{array}{l}\text { 2.A flexibilidade de acesso é um ponto positivo, pois } \\
\text { você pode acessar a qualquer hora de qualquer local. }\end{array}$ & $75 \%$ & $21 \%$ & 0 & $2 \%$ & $2 \%$ \\
\hline $\begin{array}{l}\text { 3. A experimentação remota oportuniza a todos o acesso } \\
\text { a práticas laboratoriais, considerando que algumas } \\
\text { escolas não possuem laboratórios físicos. }\end{array}$ & $69 \%$ & $21 \%$ & $10 \%$ & 0 & 0 \\
\hline $\begin{array}{l}4 . \mathrm{O} \text { experimento remoto permite um estudo mais } \\
\text { autônomo. }\end{array}$ & $55 \%$ & $36 \%$ & $7 \%$ & $2 \%$ & 0 \\
\hline $\begin{array}{l}\text { 5.Possibilidade e oportunidade de reforçar o } \\
\text { conhecimento teórico. }\end{array}$ & $69 \%$ & $31 \%$ & 0 & 0 & 0 \\
\hline $\begin{array}{l}\text { 6.Amplia as experiências de sala aula, pois incrementa } \\
\text { as atividades práticas. }\end{array}$ & $74 \%$ & $26 \%$ & 0 & 0 & 0 \\
\hline $\begin{array}{l}\text { 7.Ter o experimento remoto disponibilizado on-line é } \\
\text { um fator motivador para os estudos. }\end{array}$ & $79 \%$ & $21 \%$ & 0 & 0 & 0 \\
\hline $\begin{array}{l}\text { 8.O uso da experimentação remota para a prática de } \\
\text { ensino de física agrega qualidade ao estudo. }\end{array}$ & $72 \%$ & $26 \%$ & $2 \%$ & 0 & 0 \\
\hline $\begin{array}{l}9.0 \text { uso da experimentação remota para a prática de } \\
\text { ensino de física contribuiu para aprendizagem. }\end{array}$ & $72 \%$ & $26 \%$ & $2 \%$ & 0 & 0 \\
\hline $\begin{array}{l}\text { 10.Contribuem para a resolução das atividades e o } \\
\text { conhecimento construído a partir dos assuntos } \\
\text { trabalhados em aula. }\end{array}$ & $69 \%$ & $21 \%$ & $10 \%$ & 0 & 0 \\
\hline $\begin{array}{l}\text { 11. Respeita o ritmo de aprendizagem do estudante, uma } \\
\text { vez que pode ser acessado a qualquer momento. }\end{array}$ & $67 \%$ & $29 \%$ & $2 \%$ & $2 \%$ & 0 \\
\hline $\begin{array}{l}\text { 12. É uma importante estratégia educacional que integra } \\
\text { recursos tecnológicos, ensino aprendizagem e } \\
\text { construção do conhecimento. }\end{array}$ & $79 \%$ & $20 \%$ & $1 \%$ & 0 & 0 \\
\hline $\begin{array}{l}\text { 13. Desenvolver e disponibilizar novos experimentos } \\
\text { são importantes, visto que estes auxiliam no processo de } \\
\text { ensino aprendizagem. }\end{array}$ & $72 \%$ & $26 \%$ & $2 \%$ & 0 & 0 \\
\hline $\begin{array}{l}\text { 14. A interação entre o aluno e o experimento remoto } \\
\text { permite que o estudante participe ativamente no }\end{array}$ & $74 \%$ & $24 \%$ & $2 \%$ & 0 & 0 \\
\hline
\end{tabular}


processo de aprendizagem.

Observação: Eixo vertical número da questão e eixo horizontal grau de concordância.

Todas as afirmações apresentam grau de concordância bem elevado, ou seja, mais de $70 \%$, corroborando para uma boa aceitação do uso da experimentação remota nas aulas de física.

Em relação às questões $1,2,4,7$ e 11 podemos perceber que o experimento estar disponível on-line e ser acessado 24 horas por dia e todos os dias do ano e de qualquer lugar que tenha um computador ou celular com acesso a internet, possibilita ao aluno ajustar seu horário de estudos. Diferente do laboratório presencial que tem hora e local para ser usado, possibilita repetir várias vezes a prática experimental. Além de proporcionar ao aluno um estudo mais autônomo. As respostas se mostraram muito positivas.

Quanto às questões 5, 8, 9, 10 e 14 estas estão relacionadas com a percepção de aprendizagem dos estudantes ao usar a experimentação remota para realizar aulas práticas de física. Além de agregar qualidade ao estudo, a utilização da experimentação remota para a prática de ensino de física contribuiu para aprendizagem, mostra que o processo de aprendizagem é reforçada, uma vez que uma constante ligação é estabelecida entre experimentação e teoria como descreve Bencomo (2004). Portanto dos 40 estudantes entrevistados o percentual do grau de concordância em relação as afirmações ultrapassou os $70 \%$.

A questão 3 coloca que a experimentação remota oportuniza a todos o acesso a práticas laboratoriais, considerando que algumas escolas não possuem laboratórios físicos. Isto nos remete a realidade das escolas públicas no Brasil, onde apenas $11 \%$ possuem laboratórios de ciências, não possibilitando aos alunos destas escolas realizarem atividades práticas laboratoriais. Takahashi e Cardoso (2011) descrevem que os laboratórios de experimentação remota se apresentam como um meio de suprir a falta e a complexidade em relação ao uso de experimentos nas aulas de física. E observando o resultado da pesquisa $69 \%$ concordam totalmente, $21 \%$ concordam em parte e somente $10 \%$ ficaram neutros quanto a pergunta da pesquisa. Indo além, a questão 6 , com $74 \%$ dos alunos concordam totalmente e $26 \%$ concordam em parte que a experimentação nas aulas de física ampliam as experiências em sala de aula.

Já na questão $12,79 \%$ dos estudantes concordam totalmente e $20 \%$ concordam em parte e apenas $1 \%$ não se manifestou em relação a importância da experimentação remota como uma estratégia educacional que integra recursos tecnológicos, ensino aprendizagem e construção do conhecimento. É uma forma de trabalhar na sala de aula algo que o aluno tem muita intimidade que é a tecnologia. Mas como informa Holland (2014) é necessária a integração destas novas tecnologias através de uma criteriosa seleção de ferramentas alinhadas com as melhores práticas de ensino. Somente assim o aluno poderá ter uma aprendizagem mais eficaz.

Entretanto é necessário muitos estudos para a disseminação destes aparatos tecnológicos como os laboratórios remotos. De acordo com o percentual de aceitação da questão 13, é importante desenvolver e disponibilizar novos experimentos remotos de física, visto que estes auxiliam no processo de ensino aprendizagem.

Estes resultados fornecem um forte apoio da realidade e da autenticidade do ambiente experimental laboratório remoto e, portanto, a medida que os alunos conhecem e utilizam esta tecnologia mais resultados surgiram com uma análise mais detalhada, principalmente relacionados ao ensino e aprendizagem. 
Nesse contexto podemos dizer que "os laboratórios remotos são uma ferramenta tecnológica e pedagógica com uso crescente em todos os níveis de educação, e sua utilização generalizada é uma parte importante do seu próprio aperfeiçoamento e evolução" (FIDALGO, ANDRÉ V., et al 2013, p.2).

\section{Considerações finais}

Este trabalho descreve um projeto de inclusão de tecnológica em uma Escola de Educação Básica da Rede Pública de Ensino. A tecnologia utilizada foi o laboratório remoto com a utilização do experimento "Painel Elétrico CC". Os resultados apontam que a interação dos alunos com a experimentação remota foi positiva e válida, mostrando-se a experimentação remota "Quadro Elétrico CC" um instrumento viável para o processo de ensino e aprendizagem de física no Ensino Médio. Os alunos tiveram um alto nível de confiança ao manusear e coletar os dados do aparelho. Indo além a pesquisa identificou resultados de aprendizagem e habilidades destes alunos. $\mathrm{O}$ aparato experimental proporcionou a estes alunos aulas práticas de físicas que nas condições que a escola encontra-se não era possível.

Existem algumas limitações quanto ao uso destes laboratórios, como as dificuldades no manuseio de equipamentos tecnológicos. A prática de ensino tradicional e currículos não são apropriados para a nova estratégia de ensino-pesquisa em educação orientada - com base em experimentação remota, conforme escreve o autor (Schauer et al., 2015). Portanto, é necessário capacitar tanto professores como alunos e fazer algumas alterações curriculares onde possa ser incluso o uso de laboratórios de experimentação remota como ferramenta para auxiliar no ensino e aprendizagem.

Cabe destacar que estes experimentos remotos podem possibilitar muitos professores e alunos a acessibilidade a experiências de física sem qualquer custo e manutenção de cuidados, espaço e custos de funcionamento. Para melhores resultados poderia ser trabalhado além da experimentação remota, simulações e se possível o acesso também a laboratórios presencias.

Portanto, acredita-se que pesquisas futuras, direcionadas aos laboratórios de experimentação remota devem concentrar-se em construir e disponibilizar mais experimentos acessados remotamente voltados para o ensino de física da educação secundária. Esta será uma área crítica para o trabalho futuro, se quisermos entender como os laboratórios remotos podem ser mais eficazes no ensino e aprendizagem.

\section{Referências bibliográficas}

BENCOMO, S. D. Control learning: present and future. In: Annual Reviews In Control. Madri. abr. 2004. Disponível em: <ftp://161.24.19.221/ele/lfilipe/Lab Real Remoto/Artigos/Lab Remoto/Annual Reviews in Control.pdf>Acesso em: 22 jul. 2016.

FIDALGO, A. V. S.; ALVES. G. R.; MARQUES, M. A.; VIEGAS, M.C.; LOBO, M. C. C.; HERNÁNDEZ, U.; ZUBÍA, J. G.; GUSTAVSSON, I. Adaptação de Laboratórios Remotos a Cenários de Ensino: Casos de Estudo com VISIR e RemotElectLab. Vaep-rita, Vigo-espanha. jun. 2013. Disponível

em: $<$ http://rita.det.uvigo.es/VAEPRITA/index.php?content=Num_Pub\&idiom=Pt\&visualiza=3\&vo lumen=1\&numero=2\&articulo=10\#focoart $>$ Acesso em: 26 out. 2015.

FREIRE, Patrícia de Sá. Aumente a Qualidade e Quantidade de Suas Publicações Científicas: Manual para Elaboração de Projetos e Artigos Científicos. Curitiba: Crv, 2013. 57 p. 
HOLLAND, B. Pequenas mudanças tecnológicas: Grandes impactos na aprendizagem. Diversa - Educação Inclusiva na Prática, São Paulo, abr. 2014. Disponível em: < http://diversa.org.br/artigos/pequenas-mudancas-tecnologicas-\%C2\%96-grandes-impactos-naaprendizagem/>. Acesso em: 07 dez. 2016.

KHALIL, R. F. O uso da tecnologia de simulação na prática docente do ensino superior. In: ENCONTRO NACIONAL DE DIDÁTICA E PRÁTICAS DE ENSINO, 16., 2012, São Paulo. Anais. São Paulo: Junqueira\&marin; Editores, 2012, p. 1 - 10.

LOWE, D.; NEWCOMBE, P.; STUMPERS, B. Evaluation of the Use of Remote Laboratories for Secondary School Science Education. Research in Science Education. Austrália. Jun. 2013. Disponível em: <http://www.scopus.com/inward/record.url?eid=2-s2.084878106248\&partnerID=40\&md5=c615218fa21dcf7b9ea05979c681d324>Acesso em: 22 jul. 2016.

MOREIRA, A. C. S.; PENIDO, M. C. M. Sobre as propostas de utilização das atividades experimentais no ensino de física. In: VII ENCONTRO NACIONAL DE PESQUISA EM EDUCAÇÃ̃O EM CIÊNCIAS, 7., 2009, Florianópolis. Anais. Florianópolis: Associação Brasileira de Pesquisa em Educação em Ciências, 2009, p. 1 - 14.

PALADINI, S. Experimentação remota como suporte a ambientes de aprendizagem de física. Florianópolis: EGC/ UFSC, 2008. 89p. Dissertação de mestrado.

POZO, J. I.; CRESPO, M. Á. G. A aprendizagem e o Ensino de Ciências. Do conhecimento cotidiano ao conhecimento científico. Porto Alegre: Artmed, 2009. 296 p.

SCHAUER, F.; Ozvoldova, M.; Tkac, L.; Krbecek, M. INCLINE-The remote experimental kit for research based teaching in the class. In: INTERNATIONAL CONFERENCE ON REMOTE ENGINEERING AND VIRTUAL INSTRUMENTATION, 12th., 2015, Bangkok, Thailand. Anais. Austria: Associação Internacional de Engenharia Online (IAOE) eo Consórcio Laboratório Online (Global GOLC), 2015, p.105-108.

SCHUMACHER, E.; TAVARES, A.; SILVA, M. R.; SILVA, H. S.; DALFOVO, O.; LAVALL, I. T.; AZAMBUJA, R. A. Física Experimental Auxiliada Por Laboratório Virtual. In: XVI SIMPÓSIO NACIONAL DE ENSINO DE FÍSICA, 1., 2005. Rio de Janeiro. Anais. Rio de Janeiro: Sociedade Brasileira de Física, 2005, p. 1 - 4.

SILVA, J. B. A utilização da experimentação remota como suporte para ambientes colaborativos de aprendizagem. Florianópolis: EGC/UFSC, 2006. 196p. Tese de doutorado.

SILVA, J. B.; ROCHADEL, W.; SIMÃO, J. P.; FIDALGO, A. V. S. Uso de dispositivos móveis para acesso a Experimentos Remotos na Educação Básica. VAEP- RITA, Vigoespanha. jun 2013. Disponível em: <http://rita.det.uvigo.es/VAEPRITA/index.php?content=Num Pub\&idiom=EsJuan\&visualiza=

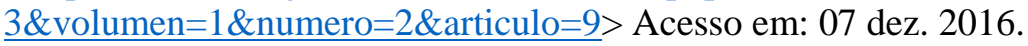

QEdu. Idealizado pela Meritt - na pessoa dos Srs. Ricardo Fritsche e Alexandre Oliveira - e pela Fundação Lemann em 2012. Apresenta dados do Censo Escolar. Disponível em: <http://www.qedu.org.br/brasil/censoescolar?year $=2014 \&$ dependence $=0 \&$ localization=0\&item;=>. Acesso em: 27 ago. 2016.

TAKAHASHI, E. K.; CARDOSO, D. C. Experimentação Remota em Atividades de Ensino Formal: um Estudo a Partir de Periódicos Qualis A. Revista Brasileira de Pesquisa em Educação em Ciências, Rio de Janeiro. set. 2011. Disponível em: <https://seer.ufmg.br/index.php/rbpec/article/view/2418/1818> Acesso em 07 dez. 2016. 